females and four males. The infants presented with fever, lethargy or irritability. The older children presented with fever and headache. Following full infection screens, all of the infants were treated with intravenous antibiotics. One patient had raised inflammatory markers and one patient had a significant cerebrospinal fluid (CSF) pleocytosis, the majority had a normal or minimally raised CSF white cell count. Eight patients had positive CSF Enterovirus PCR results and one patient had a Coxsackievirus positive serum PCR (having not undergone lumbar puncture). In three patients a prolonged course of antibiotics was discontinued following the PCR result. One patient had episodes of tachycardia with subsequent diagnosis of supraventricular tachycardia requiring treatment.

Conclusion Positive enterovirus PCR results appear to have reduced antibiotic course length, limiting unnecessary antibiotic administration, and we advocate increasing use of this investigation.

\section{PO-0257 ETIOLOGICAL STUDY OF ENTERIC VIRUSES IN ACUTE DIARRHOEA CHILDREN IN 2012, CHONGQING}

$\underline{\mathrm{H} X \mathrm{u}}, \mathrm{X}$ Tang. Department of Infectious Disease, Children'Hospital of Chongqing Medical University, Chongqing, China

\subsection{6/archdischild-2014-307384.907}

Objective Studying acute viral diarrhoea in paediatric patients in Chongqing may provide basic data and a theoretical foundation for formulating a strategy to prevent and control viral diarrhoea. Methods Diarrhoea specimens in 384 infants with suspected viral diarrhoea between January 2012 and December 2012 in Chongqing region were collected. Colloidal Gold was used for the detection of group A rotavirus (RV). RT-PCR was used for nucleic acid detection of norovirus (NV), sapovirus (SV), and astrovirus (ASV). PCR was adopted for nucleic acid detection of human adenovirus (HAdV). In addition, potential NV recombinant strains need to be identified.

Results Diarrhoea virus-positive rates were 44.27\% (170/384, RV), 21.88\% (84/384, NV), 10.16\% (39/384, HAdV), 7.81\% (30/384, ASV), and $6.77 \%(26 / 384$, SV), respectively. Mixed infection accounted for $16.15 \%(62 / 384)$ of cases. Sequencing analyses showed that GII.4, GI, ASV-1, and Ad41 genotypes were the predominant epidemic strains of NV, SV, ASV, and HAdV. A total of $5 \mathrm{NV}$ GII recombinant types were identified in this study, GII.e/GII.4 Sydney 2012, GII.7/GII.6, GII.22/GII.5, GII.12/GII.3 and GII.16/GII.13.

Conclusions RV, NV, SV, HAdV and ASV have been found to be the 5 major pathogens causing infantile viral diarrhoea in Chongqing, responsible $73.70 \%$ of the total viral diarrhoea cases. Among them, RV is the major pathogen, followed by NV.

After August 2012, the NV predominant strains of GII.4 2006 gradually changed to the recombination strains of GII.e/ GII.4 Sydney 2012, and this is the first report of the detection of GII.22/GII.5 and GII.16/GII.13 novel recombinant norovirus.

\section{PO-0258 EPIDEMIOLOGICAL AND ETIOLOGICAL CHARACTERISTIC OF HAND, FOOT, AND MOUTH DISEASE IN HOSPITAL IN CHONGQING, 2010-2013}

F Lai, H Xu. Department of Infectious Disease, Children'Hospital of Chongqing Medical University, Chongqing, China

10.1136/archdischild-2014-307384.908
Background and aim To investigated the epidemiological and etiological characteristic of Hand-Foot-Mouth disease in Chongqing, China from 2010 to 2013.

Methods Descriptive epidemiological methods were used to describe the epidemic characteristics of 3760 hospitalised cases with HFMD in Chongqing children's hospital during 2010 to 2013. The pathogens from 830 stool samples were determined by nested RT-PCR and the molecular characteristics were analysed by the phylogenetic trees.

Results There were two epidemic peaks every year during 2010 to 2013 in Chongqing. One occurred from April to July and another occurred from October to December. Most (91.22\%) of the patients were under 5 years old and $76.28 \%$ were $<3$ years of age. The ratio of male to female cases was 1.60:1. The dominant pathogens were EV71 (58.47\%) and CA16 (18.09\%). The percentage of CA16 infection cases decreased from $31.23 \%$ to $4.67 \%$ and CA6 increased from $2.11 \%$ to $16.36 \%$ from 2010 to 2013. Phylogenetic analysis indicated that EV71 isolates in Chongqing belonged to 3 lineages in subgenotype $\mathrm{C} 4 \mathrm{a}$ and CA16 isolates belonged to B1 (B1a and B1b). The molecular evolution of all the isolates from mild and severe cases were nearly identical.

Conclusions HFMD had two epidemic peaks each year in Chongqing from 2010 to 2013. The major of the patients were under 5 years old. And the percentage of males is higher than females. The pathogens are mainly EV71 C4a and CA16 B1, during 2010 to 2013. CA16 infection cases decreased while CA6 infection cases increased from 2010 to 2013.

\section{PO-0259 ANALYSIS THE VARIATION OF HEPATITIS B VIRUS GENOME IN CHILDREN FAILURE TO HB VACCINE AND THEIR MOTHERS BY HIGH-THROUGHPUT SEQUENCING}

$\underline{\mathrm{H} X \mathrm{Xu}}$. Department of Infectious Disease, Children'Hospital of Chongqing Medical University, Chongqing, China

\subsection{6/archdischild-2014-307384.909}

Objective To explore the characteristics of HBV mother-to-child transmission, and the population diversity of $\mathrm{HBV}$ in children failure to $\mathrm{HB}$ vaccine and their mothers.

Methods Eight children who developed into chronic HBV infection after receiving hepatitis $B$ vaccines and their respective mothers were enrolled into the research,the full length of HBV gene was amplified by PCR, the HBV gene was made to small fragments, different barcodes primers were attached to fragments by PCR, the 250-500 bp fragments were extracted from gel and sequenced by solexa sequencing technology. The sequencing data were analysised by statistical methods. Liver function, HBVM and HBV DNA level were determined for each patient.

Results (1) The sequences in paired mother and child could cluster well, the sequence homology between paired mother and child was beyond 99\%. (2) The significant polymorphism positions existed in whole HBV gene among the children, and $\mathrm{nt}$ 200-300 and nt 700-800 in S, the Pre C, nt 1950-2100 in C and nt 2650-2850 in P genes were the hotter sites. (3) Significant polymorphism positions could be found in every immunised child. There were 10 significant polymorphism positions in "a" determinant region among 8 pairs. aa143 only existed in children and aa124 only existed in mother. The I126T and G145R in HBsAg "a" determinant region were demonstrated in two children.

Conclusions The HBV in children are delivered from their mothers, may be the most important reason for vaccine failure. 